\title{
Nonlinear Digital Compensation for Spatial Multiplexing Systems
}

\author{
Fillipe Marques Ferreira \\ Optical Networks Group, Dept. of Electronic \& Electrical Engineering \\ University College London, \\ London WC1E 7JE, UK \\ f.ferreira@ucl.ac.uk
}

\begin{abstract}
We review the latest advances on digital backward-propagation for the compensation of inter-channel nonlinear interference in spatial- and wavelength-multiplexed systems. Different solution methods of the multimode Schrödinger equation are compared for challenging linear mode coupling and differential mode delay conditions, highlighting the significant relaxation of the step size requirements provided by the separate-channels approach.
\end{abstract}

Keywords-Digital-Back Propagation, Linear Mode Coupling, Spatial Division Multiplexing.

\section{INTRODUCTION}

Spatial Division Multiplexing (SDM) is a promising solution to overcome the capacity limit imposed by singlemode fibre (SMF) constraints [1]. SDM can be achieved over a variety of optical fibres, with few-mode fibres (FMFs) and coupled-core multi-core (CC-MCFs) offering highest spatial information density. These offer lower nonlinear coefficients and higher pumping efficiency as compared to SMF-bundles or uncoupled-core MCFs - allowing for higher spatial-density integration in transponders [2], add-drop multiplexers [3] and amplifiers [4]. However, the multitude of spatial modes introduces new impairments, namely: group delay (GD) spread [5-10] given the interplay between differential mode delay (DMD) and linear mode coupling (LMC), inter-mode nonlinear effects (IM-NL) [11-13], and mode dependent loss (MDL) [14, 15]. Among these, LMC plays a key role at decreasing the GD spread, MDL accumulation and nonlinear interactions efficiency $[16,17]$. Thus, and with practical fibres operating in all LMC regimes [18-20], LMC modelling has been under intensive research [7-10] as well as its impact on the statistics of GD, MDL and NL.

The linear impairments in SDM fibres have been successfully mitigated using a variety of digital and analogue techniques for long-haul transmission $(>1000 \mathrm{~km})$ [21-24]. However, their inter-mode nonlinearities (IM-NL) have been shown to be non-negligible $[25,26]$. And for this reason, the ability to address IM-NL through digital back propagation (DBP) has been under intensive study [27-31]. We recently shown that considerable improvements can be achieved through DBP for weak to intermediate linear coupling strength [28] and demonstrated that, in certain cases, the performance of conventional DBP may degrade with back-propagation bandwidth $[30,31]$.

In SDM-WDM systems, the compensation of the fourwave-mixing (FWM) contributions are characteristically complex - similarly to conventional WDM systems over SMFs [32-35]. The FWM process requires phase-matching in contrast to self-phase modulation (SPM) or cross-phase modulation (XPM) which are inherently phase-matched. Therefore, the strength of newly created waves (in the FWM sense) depends on the stochastic interplay between DMD and LMC (besides channel spacing, fibre dispersion, signal power, modulation format and baud rate).

In this paper, we review a separate-channel DBP implementation for SDM systems characterized by significant decorrelation, induced by DMD and LMC, between the spatial-wavelength channels. This paper is organized as follows. Section II briefly reviews DBP and the separate channels approach framework for WDM-SDM systems. Section III describes the WDM-SDM transmission setup used in the simulations. Section IV presents and analyses simulation results for conventional DBP and for separate channels DBP over a range of DMD and XT values; extending our previous results in [31]. Section V draws the final conclusions.

\section{DigitAl NONLINEAR COMPENSATION METHODS}

DBP is a nonlinear mitigation method originally proposed for SMFs [36] that compensates for the deterministic linear and nonlinear fibre impairments by numerically backpropagating the received optical field with inverted channel parameters. Back-propagation is usually implemented using the split-step Fourier method (SSFM), in our case to solve the multimode nonlinear Schrödinger equation [11-13]. However, effectiveness of this technique is reduced in the presence of random processes such as GD spread induced by DMD and $\mathrm{XT}$, like polarisation mode dispersion (PMD) in SMFs [34]. The reason being that the anti-symmetric relation between the nonlinear terms introduced by the transmission fibre and the digital nonlinear terms introduced by DBP is broken by the random evolution of the dispersion operator in the fibre (at the receiver no good assumption about this evolution can be made). This random evolution impacts FWM interactions more significantly than those resulting from XPM - since the 


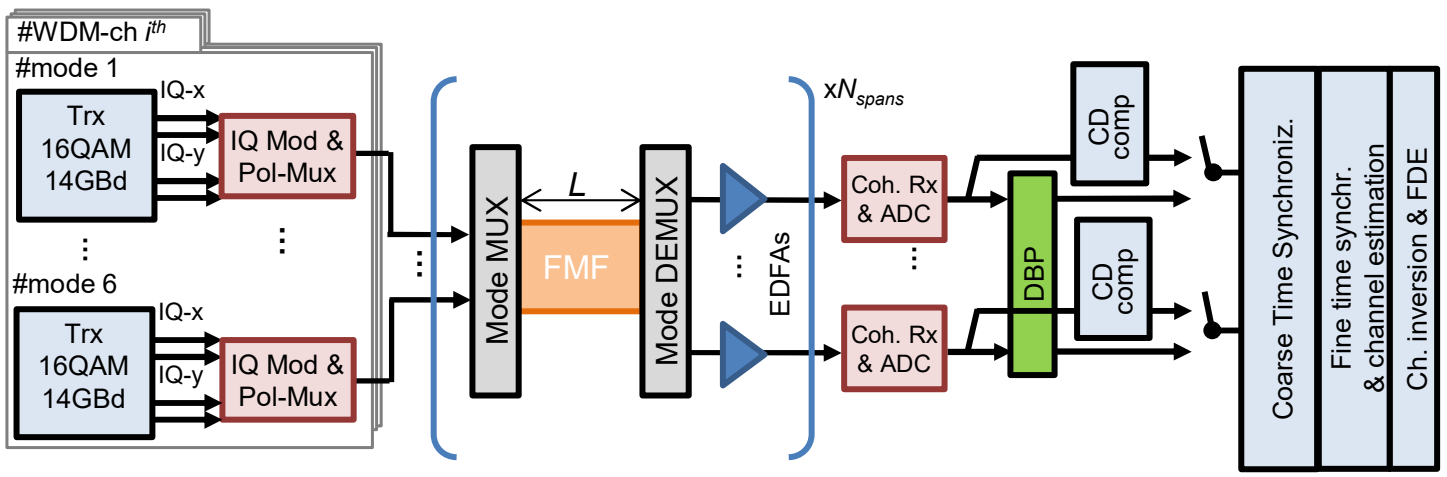

Fig. 1. Block diagram for system simulations using a fibre with 6 LP modes each with 2 orthogonal polarizations.

former requires phase-matching while the latter is inherently phase-matched. In this way, and for FMFs, conventional DBP ability to mitigate nonlinearities is further diminished by a larger DMD (than PMD) between non-degenerate modes.

SDM fibres with strong LMC and non-negligible DMD are of major practical relevance since the overall nonlinear efficiency is minimum [37] - however, this is a regime at which DBP does not perform well, as explained above. To address such problem, a simple strategy would be to have DBP attempting to correct only for SPM, intra-modal XPM and inter-modal XPM interactions - this is, to avoid DBP introducing any FWM terms. Neglecting the FWM contribution would also reduce the DBP step size requirements - XPM step requirements are relaxed by its inherent phase-matching [38, 39]. However, conventional multi-channel DBP does not allow for different nonlinear interactions to be individualised. Conventionally, a single signal envelope, $A_{i}$, is considered per mode polarisation $i$, with the product $\left|A_{i}\right|^{2} A_{i}$ leading to SPM, XPM and FWM beatings among the frequency channels contained in $A_{i}$. Instead, to remove the FWM (and intermodal FWM) components, signal propagation must be modelled using an envelope per frequency- and spatial-channel - a technique originally denominated separated-channels approach (SCA) [32]. This technique allows explicit access to all individual intra-mode and inter-mode nonlinear interactions.

Differently, and in order to actually correct for fibre FWM, gradient descent-based approaches have recently been attempted [40, 41] to learn in the digital domain a backwards modal dispersion evolution that improves DBP gain. However, computational complexity remains challenging as it scales unfavourably for shorter step-sizes. Moreover, in FMFs, larger $D M D$ values between non-degenerate modes will significantly increase the searching space.

\section{TRANSMISSION SETUP}

The WDM-SDM transmission setup considered here, with 6 linearly polarized (LP) modes $-\mathrm{LP}_{01}, \mathrm{LP}_{02}, \mathrm{LP}_{11 \mathrm{a}}, \mathrm{LP}_{11 \mathrm{~b}}$, $\mathrm{LP}_{21 \mathrm{a}}$ and $\mathrm{LP}_{21 \mathrm{~b}}-\mathrm{a}$ each with 2 orthogonal polarizations, is shown in Fig. 1. Transmission simulations consider an optical super-channel with 19 WDM channels (per mode) modulated with 14 Gbaud polarization-multiplexed 16QAM, $15 \mathrm{GHz}$ spaced; the line rate per channel is $672 \mathrm{Gbit} / \mathrm{s}$. The transmission link is composed of 7 spans of $50 \mathrm{~km}$ with a fibre loss of $0.2 \mathrm{~dB} / \mathrm{km}$ and $4.5 \mathrm{~dB}$ noise figure lumped amplification. Moreover, the effective number of bits of the analog-to-digital convertors in the receiver front-end is assumed to be 5.5 bits (consistent with state-of-the-art) - in certain cases establishing DBP upper-bound performance [42]. Further details on the transmission setup can be found in [31].

Forward transmission simulation considers a symmetric implementation of the SSFM with an adaptable step size chosen by bounding the local error [38] to be smaller than $10^{-5}$ (smaller values led to negligible change).

Two DBP methods were followed: a conventional full bandwidth back propagation as in [30] and the separate channels approach presented in [31] - in both cases a fix-step size is considered. In all cases, LMC and (residual) DMD were subsequently compensated using data-aided channel estimation and equalization. The figure of merit in the following is the minimum signal-to-noise-ratio (SNR) among the 12 polarization modes guided of the centre wavelength channel. The effective SNR is evaluated as [43] the ratio between the variance of the transmitted symbols $E\left[|X|^{2}\right]$ and the variance of the noise $E\left[|X-Y|^{2}\right], X$ and $Y$ represent the transmitted and received symbols, respectively.

\section{TRANSMISSION MODELS COMPARISON}

In the following, the performance of conventional- and SCA-DBP are presented for considering the respective optimum launch powers - around $2 \mathrm{dBm} /$ channel for the SCA-DBP and around $0 \mathrm{dBm} /$ channel for conventional DBP. Moreover, and for each method, a sufficiently short step-size as to achieve near-maximum performance is used.. The stepsize requirement for SCA-DBP was found to be 10-times longer than for conventional DBP [31]: $250 \mathrm{~m}$ for conventional DBP and $2500 \mathrm{~m}$ for SCA-DBP.

Fig. 2 shows SNR gain over linear equalization as a function of the number of back-propagated channels with $X T=-20 \mathrm{~dB} / \mathrm{m}$ and varying $D M D$. We first analyse the conventional DBP results. The figure shows that the performance of conventional DBP degrades backpropagation bandwidth - with degradation scaling with DMD and exceeding $0.5 \mathrm{~dB}$ for $D M D>10 \mathrm{ps} / \mathrm{km}$. Note that conventional DBP performance is maximum for $\sim 7$ and $\sim 3$ back-propagated channels when $D M D$ equals $2 \mathrm{ps} / \mathrm{km}$ and $5 \mathrm{ps} / \mathrm{km}$, respectively, and reducing to just one channel for $D M D \geq 10 \mathrm{ps} / \mathrm{km}$. Further results show that similar conclusions can be reached for other intermediate-strong $X T$ 


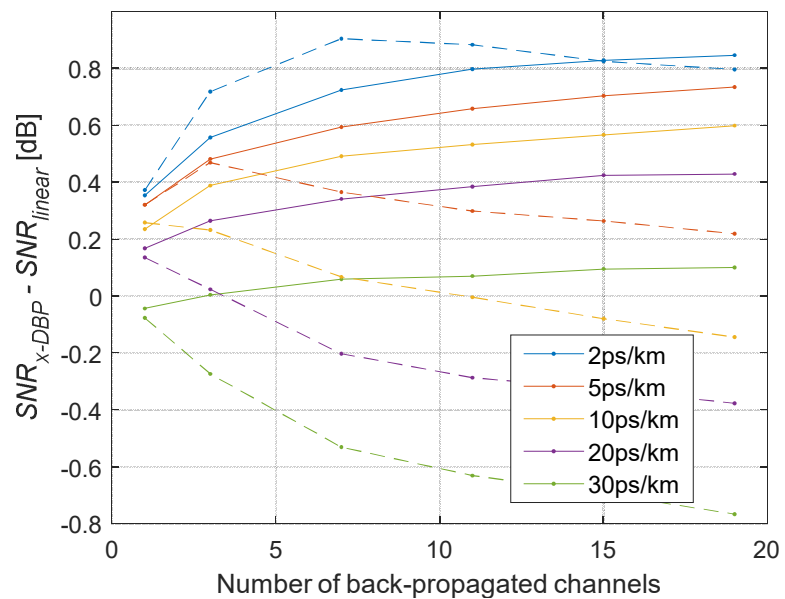

Fig. 2. $S N R$ gain over linear equalization as a function of the number of back-propagated channels $X T=-20 \mathrm{~dB} / \mathrm{m}$ and varying $D M D$, for conventional DBP (dashed lines) and SCA-DBP (full lines). Results averaged 10 repetitions.

strengths $(>-30 \mathrm{~dB} / \mathrm{m})$ - but, higher the $X T$, higher is the optimum number of back-propagated channels for a given $D M D$ value.

The results in Fig. 2 for conventional DBP seem to be at conflict with the usual expectation that higher DBP gains can be achieved for full-bandwidth propagation - however, that is not the case when the GD spread is significant (comparable or larger than the symbol period). This behaviour can be understood by noting that larger the GD spread narrower becomes the bandwidth over which channels experience similar modal dispersion evolution in the transmission fibre [44]. Therefore, attempting to DBP the outer channels will add additional nonlinear interference instead of cancelling the nonlinear interference introduced by the transmission fibre.

Conversely, Fig. 2 shows that SCA-DBP performance improves monotonically with the number of back-propagated channels. Exceeding the maximum performance of conventional DBP from very low $D M D$ values (in this case, $\geq 5 \mathrm{ps} / \mathrm{km}$ ). Note that, for $10 \mathrm{ps} / \mathrm{km}$, SCA-DBP leads to a $S N R$ improvement $>0.3 \mathrm{~dB}$ on top of the best achievable with conventional DBP - but with much loosened step-size requirement (10-times).

\section{CONCLUSION}

We have shown that a separate channels approach can be used to improve DBP performance by allowing to null the FWM terms introduced by conventional DBP - for channels that became decorrelated due to GD spread. Importantly, such an improved technique has been shown to reduce the DBP step requirements by one order of magnitude while improving maximum performance by as much as $0.3 \mathrm{~dB}$.

\section{ACKNOWLEDGMENTS}

This work was supported in part by the UKRI Future Leaders Fellowship MR/T041218/1, and in part by the Engineering and Physical Sciences Research Council Grant EP/R035342/1-TRANSNET.

To access the underlying data for this publication, see: https://doi.org/10.5522/04/14815089.

\section{REFERENCES}

[1] D. J. Richardson, J. M. Fini, and L. E. Nelson, "Space-division multiplexing in optical fibres," Nature Photonics, vol. 7, p. 354, 2013.

[2] P. J. Winzer and D. T. Neilson, "From Scaling Disparities to Integrated Parallelism: A Decathlon for a Decade," J. Lightw. Technol., vol. 35, no. 5, pp. 1099-1115, 2017.

[3] D. M. Marom et al., "Survey of photonic switching architectures and technologies in support of spatially and spectrally flexible optical networking," IEEE J. Opt. Commun. Netw., vol. 9, no. 1, pp. 1-26, 2017.

[4] H. Liu et al., "Turbulence-Resistant FSO Communication Using a FewMode Pre-Amplified Receiver," Scientific Reports, vol. 9, no. 1, p. 16247, 2019.

[5] A. Mecozzi, C. Antonelli, and M. Shtaif, "Intensity impulse response of SDM links," Opt. Express, vol. 23, no. 5, pp. 5738-43, 2015.

[6] B. Inan et al., "DSP complexity of mode-division multiplexed receivers," Opt. Express, vol. 20, no. 10, p. 10859, 2012.

[7] J. Vuong, P. Ramantanis, A. Seck, D. Bendimerad, and Y. Frignac, "Understanding discrete linear mode coupling in few-mode fiber transmission systems," in Proc. ECOC, 2011, p. Tu.5.B.2.

[8] C. Antonelli, A. Mecozzi, and M. Shtaif, "The delay spread in fibers for SDM transmission: dependence on fiber parameters and perturbations," Opt. Express, vol. 23, no. 3, pp. 2196-202, 2015.

[9] K.-P. Ho and J. M. Kahn, "Linear Propagation Effects in Mode-Division Multiplexing Systems," J. Light. Technol., vol. 32, no. 4, pp. 614-628, 2014.

[10]F. M. Ferreira, C. S. Costa, S. Sygletos, and A. D. Ellis, "Semi-Analytical Modelling of Linear Mode Coupling in Few-Mode Fibers," J. Light. Technol., vol. 35, no. 18, pp. 4011-4022, 2017.

[11]F. Poletti and P. Horak, "Description of ultrashort pulse propagation in multimode optical fibers," J. Opt. Soc. Am. B, vol. 25, no. 10, pp. 1645-1654, 2008.

[12]F. Ferreira, S. Jansen, P. Monteiro, and H. Silva, "Nonlinear semi-analytical model for simulation of few-mode fiber transmission," IEEE Photon. Technol. Lett., vol. 24, no. 4, pp. 240--242, 2012.

[13]A. Mecozzi, C. Antonelli, and M. Shtaif, "Coupled Manakov equations in multimode fibers with strongly coupled groups of modes," Opt. Express, vol. 20, no. 21, pp. 23436-23441, 2012.

[14]A. Lobato, J. Rabe, F. Ferreira, M. Kuschnerov, B. Spinnler, and B. Lankl, "Near-ML detection for MDL-impaired few-mode fiber transmission," Opt. Express, vol. 23, no. 8, pp. 9589-9601, 2015.

[15]C. Antonelli, A. Mecozzi, M. Shtaif, and P. J. Winzer, "Modeling and performance metrics of MIMO-SDM systems with different amplification schemes in the presence of mode-dependent loss," Opt. Express, vol. 23, no. 3, pp. 2203-2219, 2015.

[16]A. Lobato et al., "Impact of mode coupling on the mode-dependent loss tolerance in few-mode fiber transmission," Opt. Express, vol. 20, no. 28, p. 29776, 2012.

[17]F. M. Ferreira, C. S. Costa, S. Sygletos, and A. D. Ellis, "Nonlinear Performance of Few-Mode Fiber Links With Intermediate Coupling," $J$. Light. Technol., vol. 37, no. 3, pp. 989-999, 2019.

[18] L. Gruner-Nielsen et al., "Few Mode Transmission Fiber With Low DGD, Low Mode Coupling, and Low Loss," J. Light. Technol., vol. 30, no. 23, pp. 3693-3698, 2012.

[19]R. Ryf et al., "Space-Division Multiplexed Transmission over 4200 km 3Core Microstructured Fiber," in Proc. OFC, 2012, p. PDP5C.2.

[20]T. Hayashi et al., "Field-Deployed Multi-Core Fiber Testbed," in Proc. OECC, 2019, p. PDP3.

[21]F. Ferreira, C. Sanchez, N. Suibhne, S. Sygletos, and A. Ellis, "Nonlinear Transmission Performance in Delay-Managed Few-Mode Fiber Links with Intermediate Coupling," in Proc. OFC, 2017, p. Th2A.53. 
[22] G. Rademacher et al., "Long-Haul Transmission over Few-Mode Fibers with Space-Division Multiplexing," J. Light. Technol., vol. 36, no. 6, pp. 13821388, 2017.

[23]R. Ryf et al., "Long-Distance Transmission over Coupled-Core Multicore Fiber," in Proc. ECOC, 2016, p. Th.3.C.3.

[24]C. S. Costa, F. M. Ferreira, N. Mac Suibhne, S. Sygletos, and A. D. Ellis, "Receiver memory requirement in mode delay compensated few-mode fibre spans with intermediate coupling," in Proc. ECOC, 2016, p. Tu.1.E.4.

[25] G. Rademacher et al., "Wideband Intermodal Nonlinear Signal Processing With a Highly Nonlinear Few-Mode Fiber," IEEE J. Sel. Top. Quantum Electron, vol. 26, no. 4, pp. 1-7, 2020.

[26] G. Rademacher et al., "Investigation of Intermodal Nonlinear Signal Distortions in Few-Mode Fiber Transmission," J. Light. Technol., vol. 37, no. 4, pp. 1273-1279, 2019.

[27]R. S. Luís, B. J. Puttnam, G. Rademacher, Y. Awaji, H. Furukawa, and N. Wada, "Digital Back Propagation in Long-Haul, MIMO-Supported, Multicore Fiber Transmission," IEEE Photon. Technol. Lett., vol. 32, no. 12, pp. 730-732, 2020.

[28]F. M. Ferreira, C. S. Costa, S. Sygletos, and A. D. Ellis, "Overcoming degradation in spatial multiplexing systems with stochastic nonlinear impairments," Scientific Reports, vol. 8, no. 1, p. 17539, 2018.

[29]D. J. Elson, D. Semrau, H. Takahashi, and T. Tsuritani, "Analytical Model for Transmission Performance of Single Mode Multicore Fibre with Nonlinearity Compensation," in Proc. OECC, 2019, p. MB1.3.

[30]F. M. Ferreira, S. Sygletos, E. Sillekens, R. Killey, A. D. Ellis, and N. J. Doran, "On the Performance of Digital Back Propagation in Spatial Multiplexing Systems," J. Light. Technol., vol. 38, no. 10, pp. 2790-2798, 2020.

[31]F. M. Ferreira, E. Sillekens, B. Karanov, and R. Killey, "Digital Back Propagation via Sub-Band Processing in Spatial Multiplexing Systems," $J$. Light. Technol., vol. 39, no. 4, pp. 1020-1026, 2021.

[32] J. Leibrich and W. Rosenkranz, "Efficient numerical simulation of multichannel WDM transmission systems limited by XPM," IEEE Photon. Technol. Lett., vol. 15, no. 3, pp. 395-397, 2003.
[33]E. Mateo, F. Yaman, and G. Li, "Efficient compensation of inter-channel nonlinear effects via digital backward propagation in WDM optical transmission," Opt. Express, vol. 18, pp. 15144-54, 2010.

[34] G. Liga, T. Xu, A. Alvarado, R. I. Killey, and P. Bayvel, "On the performance of multichannel digital backpropagation in high-capacity long-haul optical transmission," Opt. Express, vol. 22, no. 24, pp. 30053-30062, 2014.

[35]C. Häger and H. D. Pfister, "Wideband Time-Domain Digital Backpropagation via Subband Processing and Deep Learning," in Proc. ECOC, 2018, p. Tu4F.4.

[36]E. Ip and J. M. Kahn, "Compensation of Dispersion and Nonlinear Impairments Using Digital Backpropagation," J. Light. Technol., vol. 26, no. 20, pp. 3416-3425, 2008.

[37]C. Antonelli, M. Shtaif, and A. Mecozzi, "Modeling of Nonlinear Propagation in Space-Division Multiplexed Fiber-Optic Transmission," $J$. Light. Technol., vol. 34, no. 1, pp. 36-54, 2016.

[38] O. V. Sinkin, R. Holzlohner, J. Zweck, and C. R. Menyuk, "Optimization of the split-step fourier method in modeling optical-fiber communications systems," J. Light. Technol., vol. 21, no. 1, pp. 61-68, 2003.

[39] A. V. T. Cartaxo, "Cross-phase modulation in intensity modulation-direct detection WDM systems with multiple optical amplifiers and dispersion compensators," J. Light. Technol., vol. 17, no. 2, pp. 178-190, 1999.

[40]C. B. Czegledi et al., "Digital backpropagation accounting for polarizationmode dispersion," Opt. Express, vol. 25, no. 3, pp. 1903-1915, 2017.

[41]E. Sillekens et al., "Time-Domain Learned Digital Back-Propagation," in IEEE SiPS, 2020, p. SS1.5.

[42]L. Galdino et al., "On the limits of digital back-propagation in the presence of transceiver noise," Opt. Express, vol. 25, no. 4, pp. 4564-4578, 2017.

[43] A. Alvarado, T. Fehenberger, B. Chen, and F. M. J. Willems, "Achievable Information Rates for Fiber Optics: Applications and Computations," $J$. Light. Technol., vol. 36, no. 2, pp. 424-439, 2018.

[44] C. Antonelli, A. Mecozzi, M. Shtaif, and P. J. Winzer, "Stokes-space analysis of modal dispersion in fibers with multiple mode transmission," Optics Express, vol. 20, no. 11, pp. 11718-11733, 2012. 